\title{
Modello de observatorio agropecuario dell cambio climático en el departamento norte de Santander, Colombia
}

\section{Model of an agricultural observatory for climate change in the northern department of Santander, Colombia}

\author{
Núñez R, Jesús; Carvajal R, Julio César; Editor Académico Prof. Ángel \\ Sol-Sánchez
}

\author{
Jesús Núñez R \\ jo.nunez@mail.udes.edu.co \\ Universidad de Santander, Colombia \\ (iD) Julio César Carvajal R \\ departamentodeeducacion@cucuta.udes \\ Universidad de Santander, Colombia \\ Editor Académico Prof. Ángel Sol-Sánchez \\ Colegio de Postgraduados, Mexico
}

\author{
Revista Iberoamericana de Bioeconomía y Cambio \\ Climático \\ Universidad Nacional Autónoma de Nicaragua, León, Nicaragua \\ ISSN-e: 2410-7980 \\ Periodicidad: Semestral \\ vol. 6, núm. 11, 2020 \\ czuniga@ct.unanleon.edu.ni
}

Recepción: 21 Marzo 2020

Aprobación: 07 Julio 2020

URL: http://portal.amelica.org/ameli/journal/394/3941758002/

DOI: https://doi.org/10.5377/ribcc.v6i11.9732

Autor de correspondencia: jo.nunez@mail.udes.edu.co

Copyright (c) 2020 Revista Iberoamericana de Bioeconomia y Cambio Climático

Esta obra está bajo una Licencia Creative Commons AtribuciónNoComercial-SinDerivar 4.0 Internacional.
Resumen: Los impactos del cambio climático en el sector agropecuario requieren de la implementación de herramientas técnicas de registro, sistematización, manejo y divulgación de la información entre los actores sociales involucrados. El Modelo de Observatorio Agropecuario del Cambio Climático en el departamento Norte de Santander (OACC), Colombia, realizado entre los años 2019 y 2020, tiene como objetivo monitorear el comportamiento del sector ante los impactos del cambio climático en 3 estaciones de estudio: planicie (220 $\mathrm{msnm})$, montaña (1.051 msnm) y páramo (2.400 msnm). El diseño del Modelo ameritó el desarrollo de las fases: a) Caracterización de las áreas agropecuarias potenciales para la instalación del OACC en el departamento; b) Determinación con los actores sociales agropecuarios de la región la pertinencia, ubicación y características del OACC; c) Diseño del modelo de Observatorio; d) Análisis de la factibilidad técnica del modelo de OACC; e) Estudio de la sostenibilidad financiera del OACC y; f) Validación del modelo con expertos en las áreas ambientales, agropecuarias y sociales. El Observatorio utilizará informaciones provenientes de las instituciones públicas y privadas y las derivadas directamente en las 3 estaciones de estudio para generar indicadores ambientales, económicos, productivos y sociales, como insumos contextualizados y pertinentes, para la formulación de políticas, planes y programas de desarrollo socioeconómico, educación, investigación, manejo de riesgos e información para los actores sociales de la entidad.

Palabras clave: Observatorio, Cambio climático, Agropecuario.

Abstract: The impacts of climate change on the agricultural sector require the implementation of technical tools for recording, systematizing, managing and disseminating information among the social actors involved. The Model of the Agricultural Observatory for Climate Change in the Department of Norte de Santander (OACC), Colombia), carried out between 2019 and 2020, aims to monitor the behaviour of the sector in the face of the impacts of climate change in three study stations: plain (220 masl), mountain (1.051 masl) and moor $(2.400 \mathrm{masl})$. The design of the Model involved the development of the following phases: a) Characterization of the potential agricultural areas for the installation of the OACC in the department; b) Determination with the agricultural social actors of the region of the relevance, 
location and characteristics of the OACC; c) Design of the Observatory model; d) Analysis of the technical feasibility of the OACC model; e) Study of the financial sustainability of the OACC and; $f$ ) Validation of the model with experts in the environmental, agricultural and social areas. The Observatory will use information from public and private institutions and those derived directly from the 3 study stations to generate environmental, economic, productive and social indicators, as contextualized and relevant inputs for the formulation of policies, plans and programs for socio-economic development, education, research, risk management and information for social actors of the entity.

Keywords: Observatory, Climate change, Agriculture.

\section{INTRODUCCIÓN}

En los últimos tiempos los Observatorios se han constituido en herramientas útiles para monitorear, analizar y difundir conocimientos de fenómenos de interés que en el transcurso del tiempo exhiben dinámicas y comportamientos variables por los diversos componentes estructurales que lo conforman. Su observación permanente y sistemática, dentro de una metodología particular diseñada para alcanzar los propósitos de estos dispositivos, genera insumos cognoscitivos pertinentes para establecer políticas y programas de intervención para disminuir sus impactos.

El Observatorio Agropecuario del Cambio Climático (OACC) en el departamento Norte de Santander se define como una herramienta científica- tecnológica de monitoreo in situ de los comportamientos de las variables climatológicas, ambientales, productivas, económicas y sociales a lo largo de los tres paisajes fisiográficos de la entidad, con el propósito de construir indicadores pertinentes que contribuyan a la toma de decisiones de los actores beneficiarios del Observatorio.

La concepción del OACC se inscribe dentro de las teorías de sistemas disipativos, incertidumbre climática y complejidad ambiental al asumir que los componentes de las variables en estudio interactúan dinámica e interdependientemente sobre territorios rurales complejos generando un conjunto de comportamientos específicos que pueden se captados in situ para predecir sus tendencias, útiles para generar acciones de mitigación, adaptación, preservación y regeneración de los tejidos afectados por el cambio climático.

La teoría de sistemas, aplicado al OACC, se afinca en la convicción de la interconectividad e independencia de las dimensiones climáticas, ambientales, productivas y sociales, al interior de sus naturalezas particulares y entre sí, exhibiendo múltiples y complejas sinergias -y rupturas- que solo pueden ser comprendidas dentro de un enfoque sistémico.

La naturaleza propia de los territorios rurales (con todos los componentes que alberga) como sistemas abiertos y complejos permite su abordaje dentro de la Teoría de Prigogine de sistemas disipativos por la emergencia de estructuras evolucionadas, autoorganizadas y coherentes derivadas de sistemas alejados del equilibrio (García, M. y Fairen, V. 1980) y, en correspondencia, como sistemas autopoéticos que se transforman en la complejidad manteniendo la identidad raigal (Maturana, 1995). En el escenario del cambio climático los ecosistemas naturales y cultivados (agro ecosistemas) reciben fuertes presiones por la variabilidad e intensidad de las temperaturas, precipitaciones y vientos que obligan a reacomodos internos

\section{Notas DE AUTOR}


en las plantas y animales, en todas sus jerarquías y ordenes, para resistir y adaptarse a las nuevas condiciones medioambientales, derivando en nuevos equilibrios y nuevas modificaciones de los ecosistemas.

Asimismo, la incertidumbre climática está asociada a la incapacidad de predecir el comportamiento climático futuro, evidenciándose confusiones por la ocurrencia paralela de fenómenos magnificados de lluvia-sequia; calor-frio; lluvia-hielo, no tradicionales en el imaginario colectivo de las sociedades humanas. En este ámbito el Panel Intergubernamental en Cambio Climático (IPCC, 2005) establece una tipología de incertidumbre climática con base a tres componentes que posibilitan su estudio: a) Impredictabilidad, por la dificultad inicial de predecir el comportamiento futuro del clima (nivel del saber cotidiano); b) Incertidumbre estructural, derivada de las limitaciones en los modelos o marcos conceptuales utilizados (nivel epistémico) y; c) Incertidumbre de valores, por la no representatividad de los datos a nivel espacial o temporal (nivel operativo).

La realidad, ahora cada vez más incierta, amerita una interpretación de la incertidumbre compleja (Soteriades, Murray-Rust, Trabucco y Metzger, 2017) en entornos medioambientales cambiantes, particularmente influenciados por el cambio climático en sus dos elementos principales: la precipitación y la temperatura. Los informes del IPCC y de la Convención Marco para el Cambio Climático de la ONU (CMNUCC) evidencian la relación entre el aumento sostenido de la temperatura desde la época preindustrial (antes del año 1.750) y la acumulación de dióxido de carbono (CO2) en la atmosfera, estando en las cercanías de superar el incremento de los $2^{\circ} \mathrm{C}$, establecido como límite superior en la COP21 realizada en Francia en 2015, y las 400 ppm de este gas en la atmosfera viabilizando a futuro escenarios climáticos (+ de $4^{\circ} \mathrm{C}$ ) de gran incertidumbre y riesgos difíciles de anticipar y planificar (Kammen, 2013).

$\mathrm{La}$ incertidumbre climática en los territorios rurales rompe los cronogramas de planificación, la gradualidad y la alternancia en la ocurrencia de los períodos secos y húmedos que permitían prever con antelación las inversiones y el desarrollo de proyectos e emprendimientos agropecuarios, observándose cambios climáticos abruptos entre sequias asfixiantes y atroces inundaciones, cuya combinación nefasta deja a su paso enormes pérdidas económicas, ecológicas y sociales por los desastres naturales que provocan los derrumbes, inundaciones, terremotos e incendios de vegetación (Febriani y Lokantara, 2017). Su génesis se deriva por la emergencia de múltiples factores exógenos interconectados de difícil reconocimiento y pronóstico (Iglesias, Quiroga, Diz y Garrote, 2011), que amerita del monitoreo y trazabilidad constante de las variables climáticas locales y la consideración de las prospectivas medioambientales en diferentes escenarios, pensando en la resiliencia futura de las obras de infraestructura y servicios agropecuarios, emprendimientos rurales y en los productores y sus familias, mediante la utilización de herramientas de análisis de decisiones y métodos de optimización dinámica para enfrentar la incertidumbre (Williams y Johnson, 2013) en sistemas de alta complejidad ambiental y humana.

$\mathrm{Al}$ asumir la existencia de una incertidumbre climática global se infiere una alta vulnerabilidad de los factores bióticos y abióticos de los ecosistemas rurales y de las poblaciones humanas por la incidencia de fenómenos climáticos extremos que deberá ser atendida para minimizar los impactos negativos en el sector agropecuario. En tiempos de cambio climático se debe comprender que las respuestas del planeta ante el calentamiento global no son lineales (causa-efecto) (Kammen, 2013), sino que se encuentran matizadas por una cascada de incertidumbre que plantea un gran desafío (Soteriades et al., 2017), para mitigar y adaptar los impactos en los sistemas naturales y sociales complejos, retos que obligan a diseñar intervenciones enmarcadas en enfoques de la complejidad ambiental y social (Supadli, Saputri y Mawengkang, 2018), la planificación de la conservación (Williams y Johnson, 2013), de la Agricultura Climáticamente Inteligente (Saj, Torquebiau, Hainzelin, Pagés y Maraux, 2017), Buenas Prácticas Agrícolas (Peixoto, Nunes, Pereira, Parreiras y Rosa, 2017), la iniciativa 4\%o para secuestrar carbono en los suelos (Chabbi, Lehmann, Ciais, Loescher, Cotrufo, Don, San Clements, Schipper, Six, Smith y Rumpei, 2017), Pagos por Servicios Ambientales (PSA) (Engel y Muller, 2016), entre muchas otras iniciativas. 
La apropiación de los actores sociales de las concepciones enmarcadas en los sistemas de comportamiento complejo y de la complejidad social (Supadli et al., 2018), características particulares de los escenarios de cambio climático, jalona los procesos dentro de un marco de la interacción de ecosistemas y sujetos en la formación prospectiva para realidades climáticas inciertas, y requieren de escenarios de mediación tecnocientífica para la consolidación de competencias en las comunidades rurales para adaptarse al clima del futuro.

En tal sentido, el objetivo de la investigación fue diseñar un Modelo de Observatorio Agropecuario que permita monitorear la trazabilidad de las huellas, impactos y tendencias del cambio climático en el sector agropecuario del departamento Norte de Santander, como eje fundamental para la producción de conocimiento contextualizado y útil que contribuya al desarrollo de políticas y acciones enfocadas a la prevención, mitigación y adaptación del sector a las variaciones medioambientales.

\section{Materiales y métodos}

\section{A. Localización}

La investigación se realizó en Colombia, en el departamento de Norte de Santander, con coordenadas $06^{\circ} 56^{\prime} 42^{\prime}$ y $09^{\circ} 18^{\prime} 01^{\prime \prime}$ de latitud norte y los $72^{\circ} 01^{\prime} 13^{\prime \prime}$ y $73^{\circ} 38^{\prime} 25^{\prime \prime}$ de longitud oeste, en los municipios El Zulia, Bochalema y Cácota de Velazco.

\section{Diseño experimental}

El área de producción agropecuaria del departamento Norte de Santander se dividió en 3 paisajes fisiográficos para estudiar el comportamiento del sector en escenarios del cambio climático: 1) Planicie aluvial; 2) Montaña y; 3) Páramo.

En cada paisaje fisiográfico se caracterizaron tres (3) municipios con potencialidades agroclimáticas e institucionales para la implementación del OACC. Estos son los municipios del Zulia (220 msnm.), Bochalema (1.051 msnm.) y Cácota de Velazco (2.400 msnm.). En cada entidad territorial seleccionada se describieron las características relacionadas con el comportamiento del clima (temperaturas, precipitaciones, vientos y humedad); condiciones edáficas (geología y tipos de suelos agrícolas); áreas de producción agrícola y pecuaria (rubros de producción y rendimientos); infraestructuras agropecuarias (vialidad, centros de apoyo a la producción, sistemas de riego, tecnologías, servicios); biodiversidad local (especies de fauna y flora); recursos hídricos (fuentes hídricas y diagnóstico de la situación actual) y; vulnerabilidad ambiental (áreas con riesgos a desastres naturales).

\section{Descripción de tratamientos}

\section{El diseño del OACC siguió las siguientes fases:}

1. Selección y caracterización de las estaciones de estudio. El Observatorio Agropecuario del Cambio Climático en el departamento Norte de Santander tendrá tres (3) estaciones de estudio, la primera (Estación Piso Cálido), estará ubicada en la zona caliente, dedicada a la producción de arroz, ganadería de carne, cacao, caña de azúcar, palma aceitera, peces y frutales; la segunda, localizada en la región de montaña media, denominada Estación Piso Templado, con vocación de uso para el cultivo del café, caña panelera, frutales, ganadería doble propósito, aves y cerdos y; la tercera, Piso Frio, ubicada en el páramo andino, consagrada al cultivo de hortalizas, flores, papa, truchas, frutales y ganadería lechera. Estas tres áreas de observación 
permitirán un monitoreo integral del comportamiento climático y agropecuario del departamento. En la figura 1, se muestra la distribución espacial de las estaciones de estudio del observatorio.

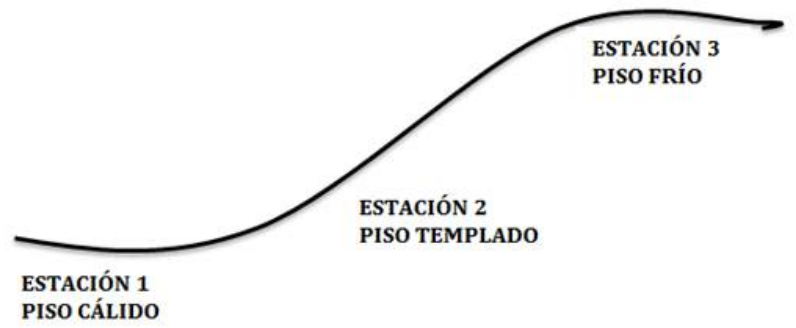

FIGURA 1

Ubicación espacial de las estaciones de estudio del Observatorio Agropecuario del Cambio Climático (OACC) en el departamento Norte de Santander Fuente: Elaboración propia (2019)

El diseño del OACC siguió las siguientes fases:

1. Selección y caracterización de las estaciones de estudio. El Observatorio Agropecuario del Cambio Climático en el departamento Norte de Santander tendrá tres (3) estaciones de estudio, la primera (Estación Piso Cálido), estará ubicada en la zona caliente, dedicada a la producción de arroz, ganadería de carne, cacao, caña de azúcar, palma aceitera, peces y frutales; la segunda, localizada en la región de montaña media, denominada Estación Piso Templado, con vocación de uso para el cultivo del café, caña panelera, frutales, ganadería doble propósito, aves y cerdos y; la tercera, Piso Frio, ubicada en el páramo andino, consagrada al cultivo de hortalizas, flores, papa, truchas, frutales y ganadería lechera. Estas tres áreas de observación permitirán un monitoreo integral del comportamiento climático y agropecuario del departamento. En la figura 1, se muestra la distribución espacial de las estaciones de estudio del observatorio.

2. Construcción del modelo de Observatorio: El OACC se fundamenta en un enfoque sistémico que involucra las múltiples interacciones de las dimensiones climáticas, ambientales, productivas y sociales sobre los territorios rurales del departamento. Estos espacios dinámicos y complejos se constituyen en los epicentros donde convergen los impactos del cambio climático sobre sus ecosistemas y poblaciones asentadas en ellos. En la representación del OACC se evidencian las interacciones entre los factores climáticos sobre los territorios generando impactos socio ambientales. Estos impactos constituyen la materia prima del Observatorio para ser registrados y sistematizados mediante procesos de minería de datos para la construcción de indicadores climáticos, productivos, económicos, ambientales y sociales, los cuales serán difundidos a través de plataformas tecnológicas a los actores involucrados con el sector agropecuario de la región (ver figura 2).

3. Estructura funcional del Observatorio: A partir de los presupuestos que configuran el modelo de OACC se delinearon los procesos del Observatorio relacionados con las estrategias de monitoreo, levantamiento de la información, minería de datos, seguimiento, evaluación, uso de plataformas tecnológicas y los actores involucrados (ver figura 3).

4. Componentes técnicos del Observatorio: Los requerimientos tecnológicos, infraestructuras y servicios de apoyo y recursos humanos se establecieron a los fines de visualizar los recursos necesarios para la implementación del OACC.

5. Análisis de los requerimientos financieros: De acuerdo a los componentes técnicos del OACC se realizó un análisis de los recursos financieros para la implementación su funcionamiento en el mediano plazo.

6. Ajustes del Modelo de OACC por consulta de expertos 


\section{Resultados}

La conformación del modelo de observatorio es una representación sistémica de los factores que confluyen en los territorios rurales, y en especial sobre las actividades agropecuarias, de tal forma que su comportamiento es la resultante de múltiples variables que actúan sobre los componentes de los sistemas naturales y humanos. En la figura 2 se presenta el diseño del modelo de OACC, una vez validado por expertos y actores locales.

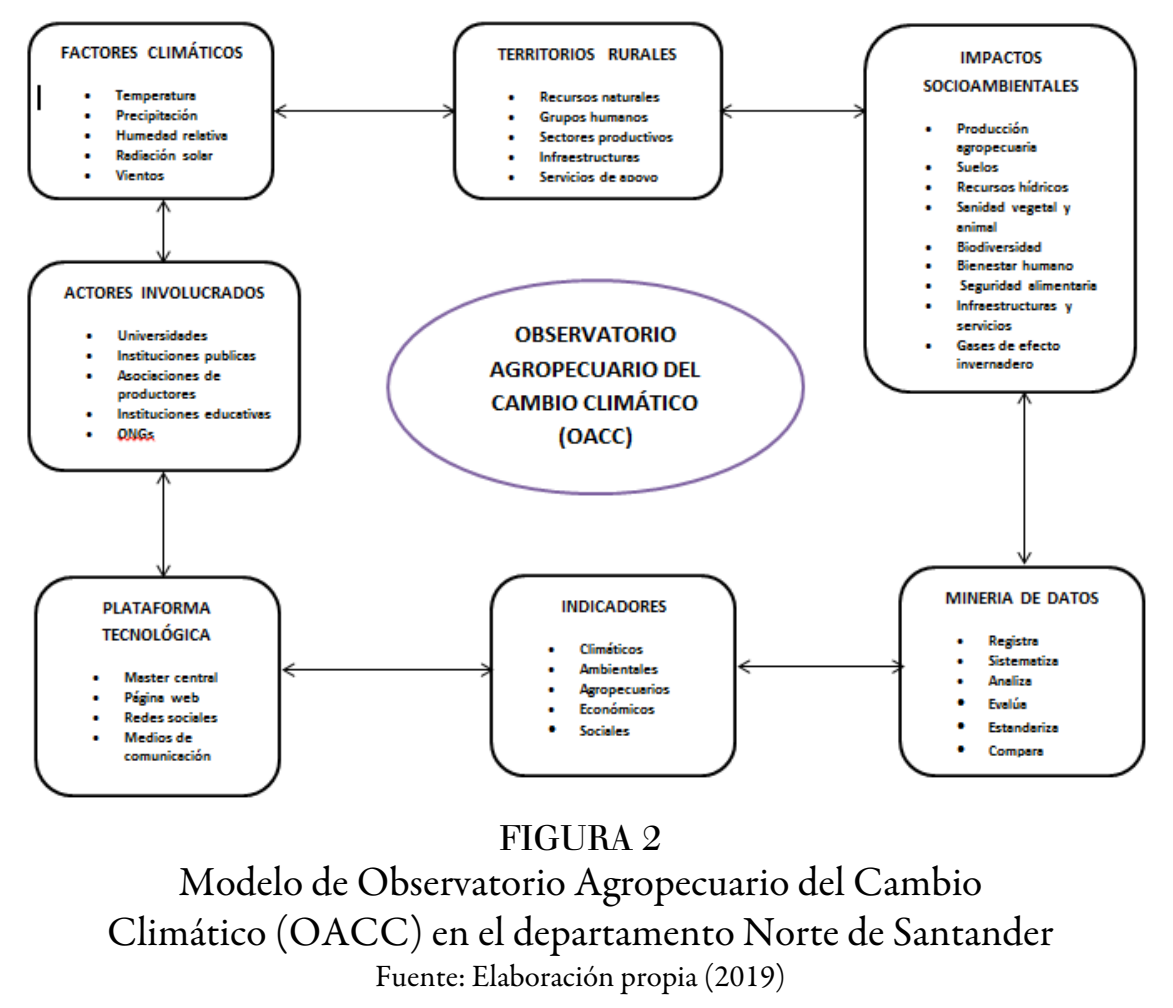

El OACC fue concebido como un sistema constituido por procesos que parten desde la interceptación de registros de información climática hasta la producción y sistematización de la información procesada como indicadores útiles y comprensibles para la toma de decisiones en los ámbitos públicos, productivos, académicos y profesionales. Un desglose de los componentes del Observatorio se presenta a continuación.

1. Factores climáticos: Es una información de primer orden para el Observatorio el registro y sistematización del comportamiento de las variables climáticas de precipitación, temperaturas, humedad relativa, vientos, radiación solar, entre otros, provenientes de instituciones medioambientales (IDEAM, CORPONOR), productivas (CENICAFE) y de captación satelital disponibles en las estaciones de estudio.

2. Territorios rurales: Se concibe como los espacios geográficos donde inciden directamente los factores climáticos y están constituidos por los recursos naturales, los grupos humanos, los sectores productivos, las infraestructuras y servicios de apoyo. Dada la gran diversidad biogeográfica del departamento Norte de Santander se ha privilegiado estratégicamente instalar 3 estaciones de observación en diferentes paisajes fisiográficos para monitorear integralmente el comportamiento del sector agropecuario de la entidad.

3. Impactos socios ambientales: Es la resultante de la acción de los factores climáticos sobre los territorios rurales y se representan como los efectos de la variabilidad climática sobre los recursos naturales y humanos. Es de interés para el OACC monitorear los impactos de los factores climáticos sobre la producción agropecuaria, biodiversidad, grupos humanos, seguridad alimentaria, suelos, recursos hídricos, salud animal, vegetal y humana, infraestructuras y servicios y la producción de gases de efecto invernadero (GEI). 
4. Minería de datos: Es el proceso de transformar los datos crudos provenientes de los registros de los factores climáticos y de los impactos socios ambientales a través de un proceso riguroso de registro, sistematización, análisis, evaluación, estandarización y comparación de la información.

5. Construcción de indicadores: Utiliza la información proveniente de la minería de datos para la construcción, validación y seguimiento de indicadores climáticos, ambientales, económicos, agropecuarios y sociales.

6. Plataforma tecnológica: Son los medios tecnológicos pertinentes para la recepción y difusión de la información procesada, convertida en indicadores, que interconecta las 3 estaciones de estudio con el campus de la UDES en la ciudad de Cúcuta. Estará conformada por una plataforma digital, master central, pagina web, redes sociales y medios de comunicación.

Actores involucrados

La operatividad del OACC se asienta sobre una estructura funcional que permite alinear los procesos de monitoreo, levantamiento de la información, minería de datos, seguimiento, evaluación, uso de plataformas tecnológicas y los actores involucrados (figura 3).

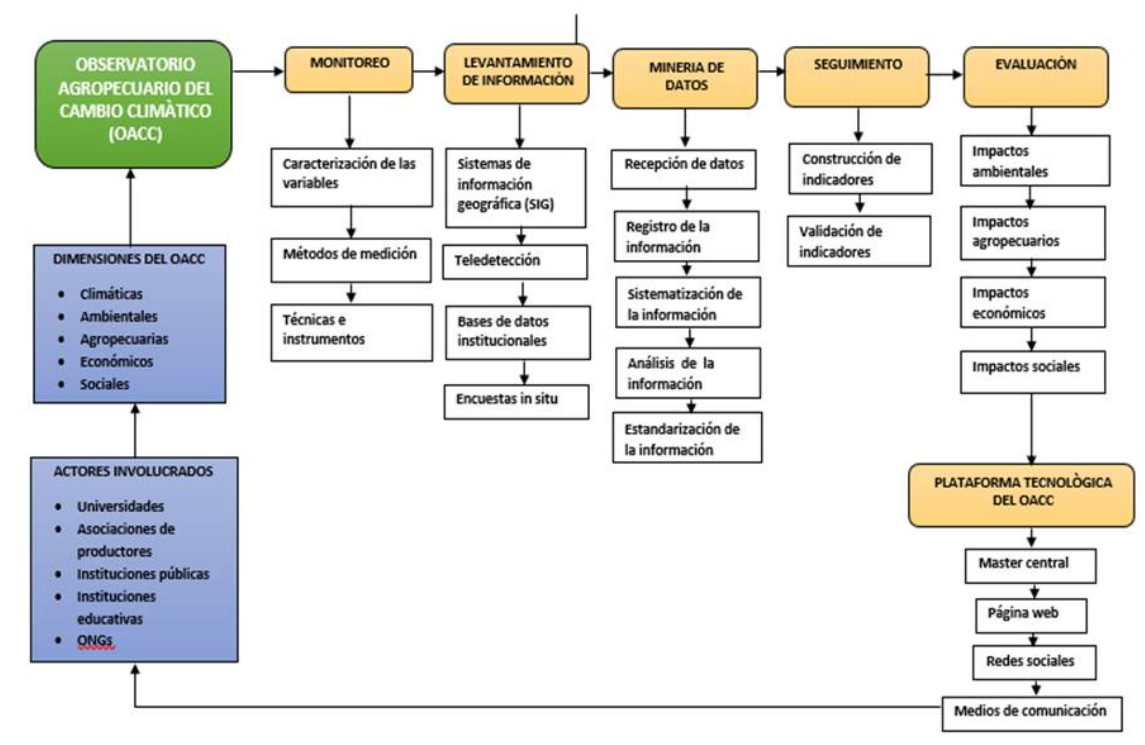

FIGURA 3

Estructura funcional del Observatorio Agropecuario del Cambio Climático (OACC) en el departamento Norte de Santander Fuente: Elaboración propia, con adaptación del modelo de Pérez (2010)

Una descripción de los procesos que implican el funcionamiento del OACC se presenta a continuación:

1. Monitoreo: Integra los métodos y técnicas de recolección de la información de campo directamente en las estaciones de estudio y provenientes de las instituciones locales, regionales, nacionales e internacionales que registran datos climáticos, productivos, económicos y sociales inherentes al departamento Norte de Santander. El monitoreo incluye la caracterización de las variables, los métodos de medición y las técnicas e instrumentos de recolección de la información.

2. Levantamiento de la información: Identifican los medios y tecnologías a utilizarse en el levantamiento de información climática, productiva, ambiental, económica y social. En el OACC es de interés la utilización del Sistema de Información Geográfica (SIG), sistemas de teledetección, bases de datos, aplicaciones digitales y las encuestas aplicadas in situ.

3. Minería de datos: En la etapa de análisis de los datos se utilizarán la estadística y las ciencias de la computación para descubrir patrones en grandes volúmenes de conjuntos de datos. Comprende los procesos de recepción, registro, sistematización, análisis y estandarización de la información. 
4. Seguimiento: Es la fase de construcción y validación de indicadores climáticos, ambientales, productivos, económicos y sociales a partir de los productos generados en la minería de datos.

5. Evaluación: Comprende la evaluación de los impactos ambientales, sociales, económicos y productivos en el sector agropecuario del departamento mediante la utilización de métodos y técnicas acreditados de evaluación de impactos.

6. Plataforma tecnológica: Se utilizará una plataforma tecnológica ágil y dinámica para la recepción y transmisión de la información entre las estaciones de estudio y la UDES y la plataforma de ésta y los usuarios del Observatorio. Se prevé la disponibilidad de un master central, pagina web, redes sociales y medios de comunicación para la divulgación de la información.

7. Actores involucrados: Conforman las instituciones que proveerán información básica para ser sistematiza por el observatorio y aquellas que serán usuarios en la utilización de los indicadores generados para apoyar la implementación de políticas públicas, programas, proyectos, investigación e intervenciones tecnocientíficas y sociales. Se identifican las asociaciones de productores agropecuarios, universidades, instituciones públicas y educativas y ONGs, entre otras.

\section{Discusión de los resultados}

La iniciativa de construir el OACC encuentra correspondencia con diversas experiencias en los ámbitos globales y locales. En Europa, La Comunidad de Trabajo de los Pirineos (CTP), creó en el 2010 el Observatorio Pirenaico del Cambio Climático (OPCC, 2010) con base el Informe presentado en el 2007 por el IPCC sobre la Evolución del Clima, quien identificó las zonas montañosas como espacios particularmente sensibles al cambio climático. El propósito de este Observatorio es "realizar un seguimiento y comprender mejor el fenómeno del cambio climático, así como realizar estudios e iniciar una reflexión para identificar las acciones necesarias para limitar los impactos y adaptarse a sus efectos" (p. s.n)

En Colombia, se instaló el Observatorio de Bosques Andinos de Antioquia (2020) bajo los auspicios del Programa Bosques Andinos, 2020 y el Pacto por los Bosques de Antioquia con el apoyo de la Agencia Suiza para del Desarrollo y la Cooperación (COSUDE), HELVETAS Swiss Intercooperation, el Consorcio para el Desarrollo Sostenible de la Ecoregión Andina (CONDESAN), el Área Metropolitana del Valle de Aburrá, el Jardín Botánico de Medellín y Mas Bosques. Entre los propósitos de este Observatorio destaca la urgencia de anticiparse a los "grandes desafíos (...) a los cambios que traerá el cambio climático para conservar estos bosques" (p.s.n).

Específicamente Observatorios enfocados a estudiar el cambio climático en el sector agropecuario son escasos en América Latina. En Costa Rica, el Observatorio Climático (2010) funciona bajo la responsabilidad del Centro Nacional de Alta Tecnología (CeNAT) y el Consejo Nacional de Rectores (CONARE), y está orientado a prestar servicios agrometeorológicos a las comunidades agrícolas con el fin de sostener la productividad agrícola y aumentar el rendimiento de los cultivos y ganado, proporcionar ayuda en la planificación y gestión de temporada de siembra y cosecha (CeNAT-CONAR, 2010).

En México, el Instituto Interamericano de Cooperación para la Agricultura (IICA, 2016) propuso, como una estrategia para integrar el sistema agroalimentario del país ante los efectos del cambio climático, la creación de un Observatorio de Gestión de Riesgos y Seguros Agropecuarios de las Américas (2016) para ofrecer "información y análisis sobre la gestión integral de riesgos que amenazan la agricultura, particularmente aquellos relacionados con el cambio climático” (p.s,n).

\section{Conclusiones}

El Observatorio Agropecuario del Cambio Climático en el departamento Norte de Santander (OACC), Colombia, es un instrumento pertinente para el monitoreo del comportamiento del sector agropecuario al generar indicadores ambientales, económicos, productivos y sociales directamente de los territorios rurales 
implicados y su triangulación con las bases de datos de las instituciones públicas y privadas relacionadas con el sector, siendo éstos insumos útiles para la toma de decisiones de los actores involucrados en la entidad.

\section{LiTERATURA CITADA}

Centro Nacional de Alta Tecnología-Consejo Nacional de Rectores CeNAT-CONARE, 2010. Programa Observatorio Climático del Área de Gestión Ambiental del Centro Nacional de Alta Tecnología. Recuperado de http://www.cenat.ac.cr/es/

Chabbi, A., Lehmann, J., Ciais, P., Loescher, H. W., Cotrufo, M.F., Don, A., San Clements, L., Schipper, J., Six, P., Smith y Rumpel, C. (2017). Aligning agriculture and climate policy, Nature climate change, 7, 307-309. doi: https://10.1038/nclimate3286

Engel, S. y Muller, A. (2016). Payments for environmental services to promote "climate - smart agriculture"? Potential and challenges, Agriculture Economics, 7(1), 173-184. doi: https://doi.org/10.1111/agec.12307

Febriani, L. y Lokantara I. G. (2017). Community participation towards the value of traditional architecture resilience, on the settlements' patters in Tenganan village, Amlapura, IOP Conf. Ser.: Earth Environ. 99(1), 1-9. doi: https://10.1088/1755-1315/99/1/012018

García, M. y Fairen, V. (1980). Estructuras disipativas. Algunas nociones básicas/1, El Basilisco, 10, 8-13. Recuperado de http://www.fgbueno.es/bas/pdf/bas11002.pdf

Iglesias, A., Quiroga, S., Diz, A. y Garrote. L. (2011). Adapting agriculture to climate change, Economia Agraria y Recursos Naturales, 11(2), 109-122. doi: https://doi.org/10.7201/earn.2011.02.05

Instituto Interamericano de Cooperación para la Agricultura, IICA, 2016. Observatorio de Gestión del Riesgo y Seguros Agropecuarios de las Américas. Recuperado de http://www.fao.org/family-farming/detail/es/ c/1054547/

Intergovernmental Panel on Climate Change (IPCC). (2005). Guidance Notes for Lead Authors of the IPCC Fourth Assessment Report on Addressing Uncertainties. Recuperado de https://wg1.ipcc.ch/publications/ supportingmaterial/uncertainty-guidance-note.pdf

Kammen, D. (2013). Complexity and interdisciplinary approaches to environmental research, Environ. Res. 8(1), 1-4. doi: https://doi.org/10.1088/1748-9326/8/1/010201

Maturana, H. (1995). La realidad: ¿Objetiva o construida? I. fundamentos biológicos de la realidad, México: Antrophos

Observatorio Pirenaico del Cambio Climático, OPCC, 2010. Recuperado de https://opcc-ctp.org/eu

Peixoto, J., Nunes, M., Baliza, D., Pereira, S., Parreiras, S. y Rosa, B.T. (2017). Cafeicultura familiar e as boas práticas agrícolas, Coffee Science, Lavras, 12(3), 365-373. doi: https://doi.org/10.25186/cs.v12i3.1298

Pérez, J. (2010). Diseño de un observatorio ambiental para el sector de curtiembres. Estudio de caso: Curtiembres de Villa Pinzón y Chocontá. (Tesis de Maestría), Universidad Nacional, Colombia. Recuperado de http://www.switchurbanwater.eu/outputs/pdfs/ W4-2_CBOG_PHD_MSc_Diseno_de_un_Observatorio_Ambiental_-_Rojas.pdf

Programa de Bosques Andinos (2020). Observatorio de bosques andinos de Antioquia. Recuperado de https:// www.observatoriobosquesantioquia.org/

Saj, S., Torquebiau, E., Hainzelin, E., Pagès, J. y Maraux, F. (2017). The way forward: An agroecological perspective for Climate-Smart Agriculture, Agriculture, Ecosystems and Environment, 250, 20-24. doi: https:// dx.doi.org/10.1016/j.agee.2017.09.003

Soteriades, A.D., Murray-Rust, D., Trabucco, A. y Metzger, M.J. (2017). Understanding global climate change scenarios through bioclimate stratification, Environmental Research Letters, 12(8), 1-10. doi: https:// doi.org/10.1088/1748-9326/aa7689 
Jesús Núñez R, et aL. Modelo de observatorio agropecuario del Cambio Climático en el dePartamento ...

Supadli, I., Saputri, A. y Mawengkang, H. (2018). Mitigating Local Natural Disaster through Social Aware Preparedness Using Complexity Approach, IOP Conf. Ser.: Mater. Sci. Eng. 300(1), 1-7. doi: https:// doi.org/10.1088/1757-899X/300/1/012050

Williams, B. K. y Johnson, F. A. (2013). Confronting dynamics and uncertainty in optimal decision making for conservation, Environ. Res, 8(2), 1-17.doi: https://doi.org/10.1088/1748-9326/8/2/025004 